\title{
STABILITY ESTIMATES BASED ON NUMERICAL RANGES WITH AN APPLICATION TO A SPECTRAL METHOD
}

\section{J. L. M. VAN DORSSELAER* and W. HUNDSDORFER}

Department of Mathematics and

Computer Science,

University of Leiden,

Niels Bohrweg 1, 2333 CA Leiden,

CWI P.O. Box 94079 1090 GB Amsterdam,

The Netherlands

dorssela@wi.leidenuniv.nl

\section{Abstract.}

This paper is concerned with the stability of numerical processes for solving initial value problems. We present a stability result which is related to a well-known theorem by von Neumann, but the requirements to be satisfied are less severe and easier to verify.

As an illustration we consider a simple convection-diffusion equation. For the spatial discretization we use a spectral collocation method (based on so-called Legendre-Gauss-Lobatto points). We show that the fully discretized numerical process is stable, provided that the temporal step size is bounded by a constant depending only on the convection-diffusion equation, the number of collocation points and the time-stepping method under consideration.

\section{AMS subject classification: $65 \mathrm{M} 12,65 \mathrm{~L} 20,65 \mathrm{M} 70$.}

Key words: stability of numerical processes, initial(-boundary) value problems, ordinary and partial differential equations, one-step and multistep methods, spectral collocation methods.

\section{Introduction.}

\subsection{The purpose of the paper.}

In this paper we consider the stability of processes

$$
u_{n}=\varphi(h A) u_{n-1}+h g_{n}, \quad n=1,2,3, \ldots,
$$

where $A$ is an $s \times s$ matrix, $g_{n} \in \mathrm{C}^{s}, h>0$ and $\varphi$ is a rational function. We write $\varphi(z)=P(z) / Q(z)$ with $P$ and $Q$ polynomials and define $\varphi(h A)=$ $P(h A) Q(h A)^{-1}$ whenever $Q(h A)$ is invertible. Starting with a vector $u_{0} \in \mathbb{C}^{s}$, the vectors $u_{n} \in \mathrm{C}^{s}$ are computed from (1.1).

Processes of the form (1.1) arise when the initial value problem

$$
\left\{\begin{array}{l}
U^{\prime}(t)=A U(t)+g(t), \quad t>0, \\
U(0)=u_{0},
\end{array}\right.
$$

* This research has been supported by the Netherlands Organization for Scientific Research (N.W.O).

Received October 1993. Revised January 1994. 
is solved with a one-step method (e.g. a Runge-Kutta method). In this case $u_{n}$ is an approximation to $U(n h), g_{n}$ is related to the vector $g(t)$, the rational function $\varphi$ is the stability function of the one-step method and the number $h$ is the step size used in the numerical integration of (1.2). The problem (1.2) itself could have been obtained from discretizing the spatial derivative(s) in a partial differential equation (accompanied with given initial- and boundary conditions). An illustration of this will be given in section 3 .

We will study the stability of process (1.1) in this paper. Suppose $\tilde{u}_{0}$ is a pertubation of the vector $u_{0}$. The vectors obtained after applying (1.1) with starting vector $\tilde{u}_{0}$ instead of $u_{0}$ will be denoted by $\tilde{u}_{n}$. With $v_{n}=\tilde{u}_{n}-u_{n}$ we arrive at $v_{n}=\varphi(h A) v_{n-1}$. Since $v_{n}=\varphi(h A)^{n} v_{0}$, it is important in the stability analysis of (1.1) that one is capable of predicting the growth of

$$
\left\|\varphi(h A)^{n}\right\|
$$

where $\|\cdot\|$ stands for some appropriate matrix norm. In this paper we restrict ourselves to norms $\|\cdot\|$ generated by an inner product norm on $\mathbf{C}^{s}$.

In this paper we shall present a sufficient condition on $h A$ such that $\left\|\varphi(h A)^{n}\right\|$ $\leq 3$. Our estimate is essentially based on material presented in Sz.-Nagy and Foiaş [15], [16]. This result is related to a well-known theorem due to von Neumann and recent investigations by Lenferink and Spijker [7], Lubich and Nevanlinna [8], Reddy and Trefethen [13]. A similar stability estimate will be presented for multistep discretizations of (1.2).

To illustrate our result we consider a convection-diffusion equation in which we discretize the spatial derivatives with a spectral collocation method (based on the so-called Legendre-Gauss-Lobatto points). We provide a sufficient condition on the step size $h$ such that (1.1) is stable. As far as we know such a result for spectral collocation methods has only been obtained for hyperbolic equations (see e.g. [4], [12]).

\subsection{Contents of the paper.}

In subsection 2.1 we introduce some notations and definitions which will be used in the remainder of the paper. In subsection 2.2 we present the results providing an upper bound for $\left\|\varphi(h A)^{n}\right\|$. Along with a theorem by von Neumann, we present a related stability estimate (Theorem 2.4 ) based on so-called numerical ranges, as well as other results. Analogous stability estimates for multistep methods will be presented in subsection 2.3 .

In section 3 we will discuss an application of our theory to a spectral collocation discretization of a convection-diffusion equation. We show that the matrix $A$ obtained after the spatial discretization of the partial differential equation satisfies the requirements of Theorem 2.4. Further we determine an $H>0$ such that (1.1) is stable whenever $0<h \leq H$. This threshold value $H$ depends only on the parameter in the convection-diffusion equation, the number of collocation points and the one-step method under consideration.

In this paper the rational function $\varphi$ always stands for the stability function

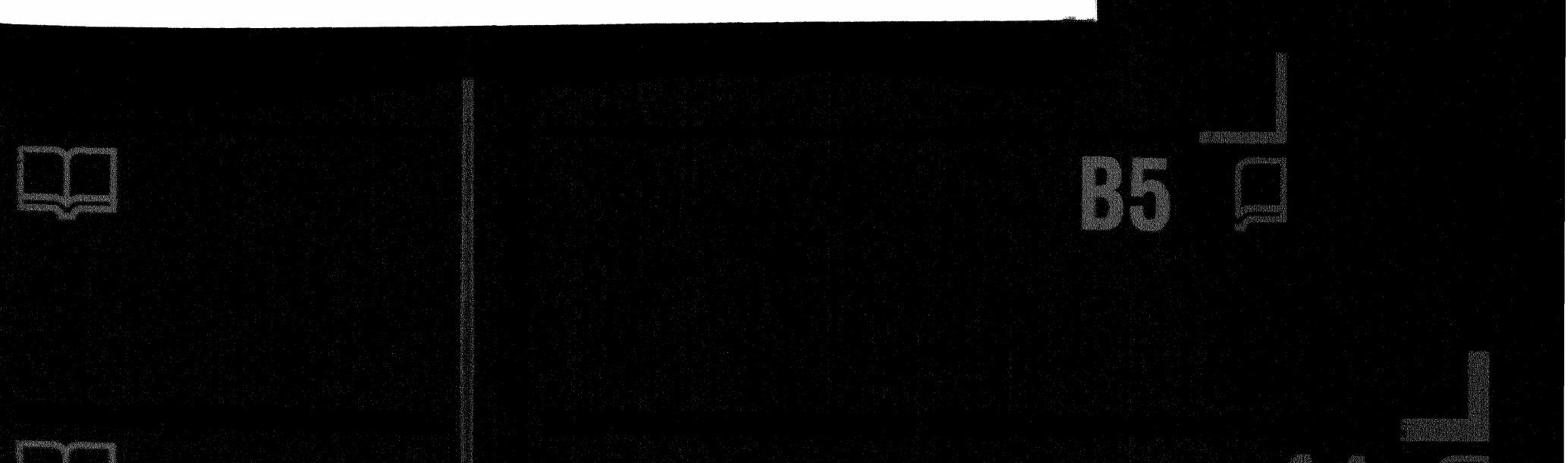


of a one-step method. We sometimes identify one-step methods with their stability function.

\section{Stability estimates.}

\subsection{Notations and definitions.}

In this subsection we will present some notations and definitions which will be used in the remainder of this paper.

In the following $I$ stands for the $s \times s$ identity matrix. An arbitrary inner product on the space $\mathrm{C}^{s}$ and the corresponding vector norm will be denoted by $\langle x, y\rangle$ and $|x|=\sqrt{\langle x, x\rangle}$ respectively $\left(x, y \in \mathbf{C}^{s}\right)$. For an $s \times s$ matrix $B$ we define the induced matrix norm $\|B\|=\sup \left\{|B x|: x \in \mathbf{C}^{s}\right.$ with $\left.|x|=1\right\}$ and the numerical range (with respect to the inner product $\langle\cdot, \cdot\rangle$ )

$$
F(B)=\left\{\zeta: \zeta \in \mathbf{C} \text { and } \zeta=\langle B x, x\rangle \text { with } x \in \mathbf{C}^{s},\langle x, x\rangle=1\right\} .
$$

The Euclidean inner product will be written as $\langle x, y\rangle_{2}=y^{*} x\left(y^{*}\right.$ is the Hermitian adjoint of $y$ ). Its induced matrix norm and numerical range are denoted by $\|\cdot\|_{2}$ and $F_{2}(\cdot)$, respectively. The numerical range $F_{2}(B)$ is sometimes called the field of values of the matrix $B$, and for that reason we denote numerical ranges with $F(\cdot)$.

The complex left half plane is denoted by $\mathbf{C}^{-}=\{\zeta: \zeta \in \mathbf{C}$ with $\operatorname{Re} \zeta \leq 0\}$ and

$$
D[c, r]=\{\zeta: \zeta \in \mathbf{C} \text { with }|\zeta-c| \leq r\}
$$

stands for a disk in the complex plane with centre $c$ and radius $r$.

For a one-step method with stability function $\varphi$ the stability region is defined as

$$
S=\{\zeta: \zeta \in \mathbf{C} \text { with }|\varphi(\zeta)| \leq 1\} .
$$

Further we introduce the stability radius $R$ of the one-step method

$$
R=\sup \{r: r \geq 0 \text { and } D[-r, r] \subset \mathrm{S}\} .
$$

We note that $R>0$ for each consistent one-step method (cf. e.g. [9, p. 517]). For several polynomials $\varphi$ satisfying $\varphi(0)=\varphi^{\prime}(0)=1$ the stability radius has been computed in [9].

\subsection{Stability estimates for one-step methods.}

Unless otherwise stated, all results in section 2 are valid for norms and numerical ranges induced by arbitrary inner products $\langle\cdot, \cdot\rangle$.

The following theorem can be proved by using results due to von Neumann (see e.g. [14, section 153]). Then

THEOREM 2.1. Let $r$ and $h A$ be such that $\|h A+r I\| \leq r$ and $D[-r, r] \subset S$.

$$
\left\|\varphi(h A)^{n}\right\| \leq 1 \text { for } n=1,2,3, \ldots
$$


This is a well-known result which gives a reliable stability estimate for the process (1.1). The only disadvantage is that the restriction $\|h A+r I\| \leq r$ may be rather severe or difficult to verify. However for some special rational functions $\varphi$ it is possible to relax the requirements on $h A$ in Theorem 2.1; the following result is well-known (see e.g. [5, p. 179]) and can be proved by using Theorem 2.1 .

THEOREM 2.2. Let $F(h A) \subset \mathrm{C}^{-} \subset S$. Then

$$
\left\|\varphi(h A)^{n}\right\| \leq 1 \text { for } n=1,2,3, \ldots .
$$

Theorem 2.2 can only be used for a special class of implicit one-step methods (the so-called $A$-stable methods, see e.g. [5]).

For the (explicit) forward Euler method we have the following result.

THEOREM 2.3. Let $\varphi(z)=1+z$ and $F(h A) \subset S$. Then

$$
\left\|\varphi(h A)^{n}\right\| \leq 2 \text { for } n=1,2,3, \ldots
$$

Proof. $F(h A) \subset S=D[-1,1]$ implies that $F(\varphi(h A)) \subset D[0,1]$. Theorem 2.3 follows from the fact that $F(B) \subset D[0,1]$ implies $\left\|B^{n}\right\| \leq 2$ for $n=1,2$, $3, \ldots$ (see e.g. [15, p. 17]).

In view of Theorems 2.2 and 2.3 one may ask whether the implication ' $F(h A)$ $\subset S$ implies that $\left\|\varphi(h A)^{n}\right\|$ is uniformly bounded' holds for arbitrary functions $\varphi$. A first attempt to prove this might be based on generalizing the proof of Theorem 2.3 by showing that $F(h A) \subset S$ implies $F(\varphi(h A)) \subset D[0,1]$. Unfortunately, the latter implication does not hold in general as the following counterexample shows. Let

$$
A=\left(\begin{array}{cc}
-1 & 2 \\
0 & -1
\end{array}\right)
$$

and $\varphi(z)=1+z-z^{2}$. It is easy to see that $F_{2}(A)=D[-1,1]$. Hence there exists a $h>0$ such that $F_{2}(h A)=D[-h, h] \subset S$. On the other hand, $F_{2}(\varphi(h A))$ $=D\left[1-h-h^{2}, h+2 h^{2}\right]$ so that $F_{2}(\varphi(h A)) \not \subset D[0,1]$ for all $h>0$.

In spite of this counterexample it is possible to generalize Theorem 2.3 in a certain sense to arbitrary rational functions $\varphi$.

THEOREM 2.4. Let $r$ and $h A$ be such that $F(h A) \subset D[-r, r] \subset S$. Then

$$
\left\|\varphi(h A)^{n}\right\| \leq 3 \text { for } n=1,2,3, \ldots
$$

Proof. In Sz.-Nagy and Foiaş [15], [16] the class of linear operators $C_{\rho}$, consisting of unitary $\rho$-dilations, has been introduced and it was shown that

$$
\|\psi(B)\| \leq \max _{|z| \leq 1}|\rho \psi(z)+(1-\rho) \psi(0)|
$$

for any $B \in C_{\rho}$ and all rational functions $\psi$ which are analytic on the closed unit 
disk. According to a theorem of Berger and Halmos (Proposition I.11.2 in [16], see also p. 55 loc. cit.) it holds that

$$
B \in C_{2} \Leftrightarrow F(B) \subset D[0,1] .
$$

Now, let $n \geq 1$ be fixed and define $\psi(z)=\varphi(r z-r)^{n}, B=r^{-1}(h A+r I)$. Then $\psi(B)=\varphi(h A)^{n}$ and $F(B) \subset D[0,1]$. From the above it follows that

$$
\left\|\varphi(h A)^{n}\right\| \leq \max _{z \in D[-r, r]}\left|2 \varphi(z)^{n}-\varphi(-r)^{n}\right| \leq 3 .
$$

This theorem can be viewed as a generalization to Theorem 2.3 ; in fact, in the last inequality of the proof the factor 2 can be reobtained in case $\varphi(z)=1+$ $z$ and $r=1$.

Theorem 2.4 is also related to Theorem 2.1 , but the requirements to be satisfied are less severe; it is easy to see that $\|h A+r I\| \leq r$ implies $F(h A) \subset$ $D[-r, r]$ but the converse is in general not true: with $A$ as in (2.1) we have $F_{2}(h A) \subset D[-r, r]$ for $r \geq h$ while $\|h A+r I\|_{2} \leq r$ cannot be fulfilled for any positive number $r$.

We note that Theorem 2.1 can be proved in the same way as Theorem 2.4. The class $C_{1}$ satisfies $C_{1}=\{B:\|B\| \leq 1\}$ (see e.g. [15], [16]) and Theorem 2.1 follows from the proof of Theorem 2.4 with $\rho=1$.

Theorem 2.4 can be generalized to the case of variable step sizes (Ch. Lubich, private communication to the authors).

REMARK 2.5. Recently Lenferink and Spijker [7], Lubich and Nevanlinna [8], Reddy and Trefethen [13] and others have provided an upper bound for $\left\|\varphi(h A)^{n}\right\|$, based on so-called resolvent conditions (cf. e.g. [2, subsection 3.2]). In these papers they consider the resolvent condition

(2.2) $\zeta I-h A$ is invertible and $\left\|(\zeta I-h A)^{-1}\right\| \leq K \mathrm{~d}(\zeta, V)^{-1}$ for all $\zeta \notin V$

where $V$ is a closed subset of the stability region $S, K \geq 1$ is a constant and $\mathrm{d}(\zeta, V)$ denotes the distance from $\zeta$ to $V$. Under condition (2.2) and some technical restrictions on the function $\varphi$ and the set $V$ they provide upper bounds for $\left\|\varphi(h A)^{n}\right\|$. Unfortunately, these upper bounds are not uniformly in $n$ and $s$ (the order of the matrix $h A$ ), unless $V$ is required to be a subset of the wedge $W(\alpha)=\{\zeta: \zeta=0$ or $|\arg \zeta-\pi| \leq \alpha\}(\alpha \in[0, \pi / 2))$ (see e.g. [7, Lemma 3.1]). These results based on resolvent conditions are related to Theorems 2.2-2.4 since (2.2) is satisfied with $V=F(h A)$ and $K=1$ (this follows from [6, Theorem 2.1] (cf. e.g. [2, subsection 4.2])). In order to obtain uniform boundedness of $\left\|\varphi(h A)^{n}\right\|$ from the results in loc. cit., we need that $F(h A)$ lies in a wedge $W(\alpha)$.

\subsection{Generalization to multistep methods.}

Similar as in Theorem 2.4 a stability estimate can be obtained for multistep methods. Discretization of (1.2) with a $k$-step method gives a recursion of the type 


$$
u_{n}=\sum_{j=0}^{k-1} \varphi_{j}(h A) u_{n-k+j}+h g_{n}, \quad n=k, k+1, k+2, \ldots
$$

with starting vectors $u_{0}, u_{1}, \ldots, u_{k-1}$ and with rational functions $\varphi_{0}, \varphi_{1}, \ldots$, $\varphi_{k-1}$ determined by the specific method.

In order to study the stability of (2.3) we consider perturbed starting vectors $\tilde{u}_{0}, \tilde{u}_{1}, \ldots, \tilde{u}_{k-1}$. The vectors obtained by applying (2.3) with these starting vectors are denoted by $\tilde{u}_{n}$ and we let $v_{n}=\tilde{u}_{n}-u_{n}$. Further, it is convenient to introduce $V_{n}=\left(v_{n}^{\mathrm{T}}, v_{n-1}^{\mathrm{T}}, \ldots, v_{n-k+1}^{\mathrm{T}}\right)^{\mathrm{T}} \in \mathbf{C}^{k s}$. These vectors satisfy

$$
V_{n}=C(h A) V_{n-1} \quad \text { for } n \geq k \text {, }
$$

where $C(h A)$ is the $k s \times k s$ companion block-matrix

$$
C(h A)=\left(\begin{array}{ccccc}
\varphi_{k-1}(h A) & \varphi_{k-2}(h A) & \ldots & \ldots & \varphi_{0}(h A) \\
I & O & \ldots & \ldots & O \\
O & I & O & \ldots & O \\
\vdots & \ddots & \ddots & \ddots & \vdots \\
O & \ldots & O & I & O
\end{array}\right) .
$$

The stability region of the method can be characterized as

$$
S=\{\zeta: \zeta \in \mathbf{C} \text { with } C(\zeta) \text { power bounded }\} \text {. }
$$

The power boundedness of $C(\zeta)$ can be expressed in terms of the eigenvalues of $C(\zeta)$ to arrive at the usual root condition, as given, for instance, in [5, p. 257].

From (2.4) it follows that $V_{n}=C(h A)^{n-k+1} V_{k-1}$. In terms of the $v_{n}$ we obtain

$$
v_{n}=\sum_{j=0}^{k-1} \psi_{n, j}(h A) v_{j} \quad \text { for } n \geq k
$$

with rational functions $\psi_{n, j}(\zeta)$ given by the first row of $\mathrm{C}(\zeta)^{n-k+1}$. So, stability of the process $(2.3)$ is determined by upper bounds for $\left\|\psi_{n, j}(h A)\right\|$.

Consider $r \geq 0$ such that $D[-r, r] \subset S$. As in [5, pp. 351-352] it can be seen that there exists a finite $M>0$ such that

$$
\left|\psi_{n, j}(z)\right| \leq M \text { for all } z \in D[-r, r] \text { and } n \geq k, \quad 0 \leq j \leq k-1 \text {. }
$$

In the same way as in the proof of Theorem 2.4 , with $\psi$ taken as $\psi_{n, j}$, we thus obtain the following result.

THEOREM 2.6. Suppose $F(h A) \subset D[-r, r] \subset S$ and let $M$ be as in (2.6). Then

$$
\left\|\psi_{n, j}(h A)\right\| \leq 3 M \text { for } n \geq k, \quad 0 \leq j \leq k-1 .
$$

Although there are linear multistep methods which do not satisfy $D[-r, r] \subset$ $S$ for some $r>0$ (the explicit midpoint rule, for example), for most methods such $r>0$ does exist; see e.g. [5, pp. 259, 262].

A generalization of Theorems 2.1 and 2.2 to multistep methods has been 
obtained in [11] (cf. also [5, p. 356]). In that paper the power boundedness of the matrix (2.4b) has been proved under the same restrictions on $h A$ as in the corresponding theorems for one-step methods.

Stability estimates for (linear) multistep methods based on resolvent conditions of type (2.2) have been proved by [8], [12], [13] and others.

\section{An application to Theorem 2.4.}

In order to illustrate Theorem 2.4 we consider the following convectiondiffusion equation

$$
\left\{\begin{array}{l}
u_{t}(x, t)+u_{x}(x, t)=\nu u_{x x}(x, t)+f(x, t), \quad-1<x<1, \quad t>0 \\
u(-1, t)=a(t), u(1, t)=b(t), u(x, 0)=c(x)
\end{array}\right.
$$

Here $\nu$ is a positive constant and $a, b, c$ and $f$ are smooth real-valued functions.

Following the method of lines approach, we first discretize the spatial derivatives in (3.1). The spatial discretization we consider is based on a so-called spectral collocation or pseudospectral method (see e.g. [1]). For a positive integer $N$ we introduce the so-called collocation points

$$
1=x_{0}>x_{1}>\ldots>x_{N}=-1 \text {. }
$$

The Lagrange polynomials $L_{j}$ of degree $N$ with respect to the points (3.2) are defined by $L_{j}\left(x_{j}\right)=1$ and $L_{j}\left(x_{i}\right)=0$ for $i, j=0,1,2, \ldots, N$ and $i \neq j$. For each $t$ and $x$ we approximate the true solution to (3.1) by

$$
u_{N}(x, t)=\sum_{j=1}^{N-1} U_{j}(t) L_{j}(x)+b(t) L_{0}(x)+a(t) L_{N}(x) .
$$

Note that $u_{N}$ satisfies the boundary conditions in (3.1). The unknowns $U_{j}(t)$ are determined by requiring that $u_{N}$ satisfies the differential equation and initial condition in (3.1) at the collocation points $x_{1}, x_{2}, \ldots, x_{N-1}$. With the notation $U(t)=\left(U_{1}(t), U_{2}(t), \ldots, U_{N-1}(t)\right)^{\mathrm{T}}$ this leads to the initial value problem (1.2) with dimension $N-1$, where $A$ is given by

$A=-D^{(1)}+\nu D^{(2)}$ with $D_{i j}^{(1)}=\mathrm{L}_{j}^{\prime}\left(x_{i}\right), \mathrm{D}_{i j}^{(2)}=\mathrm{L}_{j}^{\prime \prime}\left(x_{i}\right)$ for $i, j=1,2, \ldots, N-1$,

and with $u_{0}$ depending on $c$, and $g(t)$ depending on the functions $a, b$ and $f$. After discretizing (1.2) with a one-step method one arrives at the problem (1.1).

In this paper the collocation points (3.2) under consideration are the socalled Legendre-Gauss-Lobatto points (see e.g. [1, subsection 2.3.1]). In order to apply Theorem 2.4 we will need an inner product. Define for $y, z \in \mathbf{C}^{N-1}$ the inner product

$$
\langle y, z\rangle_{N}=\sum_{j=1}^{N-1} y_{j} \bar{z}_{j} w_{j},
$$

where the numbers $w_{j}>0$ are the so-called Legendre-Gauss-Lobatto weights. The related quadrature rule 


$$
\int_{-1}^{1} q(x) \mathrm{d} x \approx \sum_{j=0}^{N} q\left(x_{j}\right) w_{j}
$$

is exact for polynomials $q$ of degree $\leq 2 N-1$ (see e.g. [1, subsection 2.2]). The vector norm, matrix norm and numerical range corresponding to $\langle\cdot, \cdot\rangle_{N}$ will be denoted by $|\cdot|_{N},\|\cdot\|_{N}$ and $F_{N}(\cdot)$ respectively. Further we introduce the numbers

$$
\alpha_{1}=\max _{\zeta \in F_{N}\left(D^{(1)}\right)}|\zeta| \text { and } \alpha_{2}=\max _{\zeta \in F_{N}\left(D^{(2)}\right)}|\zeta| \text {. }
$$

THEOREM 3.1. For each $\nu>0$ and $N \geq 2$ there exist a number $r>0$ such that $F_{N}(A) \subset D[-r, r]$. Moreover $r$ can be chosen such that

$$
r \leq r_{1}
$$

where $r_{1}$ is given by

$$
r_{1}=\frac{1}{2 \nu} \max \left[\nu^{2} \alpha_{1}^{2}+1, \nu^{2} \alpha_{2}+\left(\alpha_{1}^{2} / \alpha_{2}\right)\right]
$$

Proof. For $y \in \mathbf{C}^{N-1},\langle y, y\rangle_{N}=1$ there exists exactly one polynomial $p$ of degree $\leq N$ satisfying $p\left(x_{j}\right)=y_{j}(j=1,2, \ldots, N-1)$ and $p(-1)=p(1)=0$. From the property $p(-1)=p(1)=0$, the fact that the quadrature rule (3.4) is exact for polynomials $q$ of degree $\leq 2 N-1$ and some partial integration it follows that

$$
\begin{aligned}
\left\langle D^{(1)} y, y\right\rangle_{N} & =\sum_{j=1}^{N-1} p^{\prime}\left(x_{j}\right) \overline{p\left(x_{j}\right)} w_{j}=\int_{-1}^{1} p^{\prime}(x) \overline{p(x)} \mathrm{d} x=-\int_{-1}^{1} \overline{p^{\prime}(x)} p(x) \mathrm{d} x, \\
\left\langle D^{(2)} y, y\right\rangle_{N} & =\sum_{j=1}^{N-1} p^{\prime \prime}\left(x_{j}\right) \overline{p\left(x_{j}\right)} w_{j}=\int_{-1}^{1} p^{\prime \prime}(x) \overline{p(x)} \mathrm{d} x=-\int_{-1}^{1}\left|p^{\prime}(x)\right|^{2} \mathrm{~d} x \\
& =-\sum_{j=0}^{N}\left|p^{\prime}\left(x_{j}\right)\right|^{2} w_{j}=-\left|D^{(1)} y\right|_{N}^{2}-w_{0}\left|p^{\prime}\left(x_{0}\right)\right|^{2}-w_{N}\left|p^{\prime}\left(x_{N}\right)\right|^{2} .
\end{aligned}
$$

So we can conclude that $\left\langle D^{(1)} y, y\right\rangle_{N}$ is purely imaginary, $\left\langle D^{(2)} y, y\right\rangle_{N}$ is real and negative, and

$$
\left|\left\langle D^{(1)} y, y\right\rangle_{N}\right|^{2} \leq\left|D^{(1)} y\right|_{N}^{2} \leq\left|\left\langle D^{(2)} y, y\right\rangle_{N}\right| .
$$

Let $\xi=\left|\left\langle D^{(2)} y, y\right\rangle_{N}\right|, \eta=\left\langle D^{(1)} y, y\right\rangle_{N}$. We have

$$
\left|\langle A y, y\rangle_{N}+r\right|^{2}=(-\nu \xi+r)^{2}+|\eta|^{2} \leq(-\nu \xi+r)^{2}+\min \left(\xi, \alpha_{1}^{2}\right) .
$$

By some straightforward calculations it follows that $F_{N}(A) \subset D[-r, r]$ is satisfied when $r \geq r_{1}$.

We note that the idea of using Gauss-Lobatto quadrature in order to obtain useful results for $\left\langle D^{(2)} y, y\right\rangle_{N}$ is due to Gottlieb [3] (see also [1, subsection 11.4.2]).

From Theorem 3.1 it follows that (1.1) is stable when $h \leq R / r_{1}$. In order to 

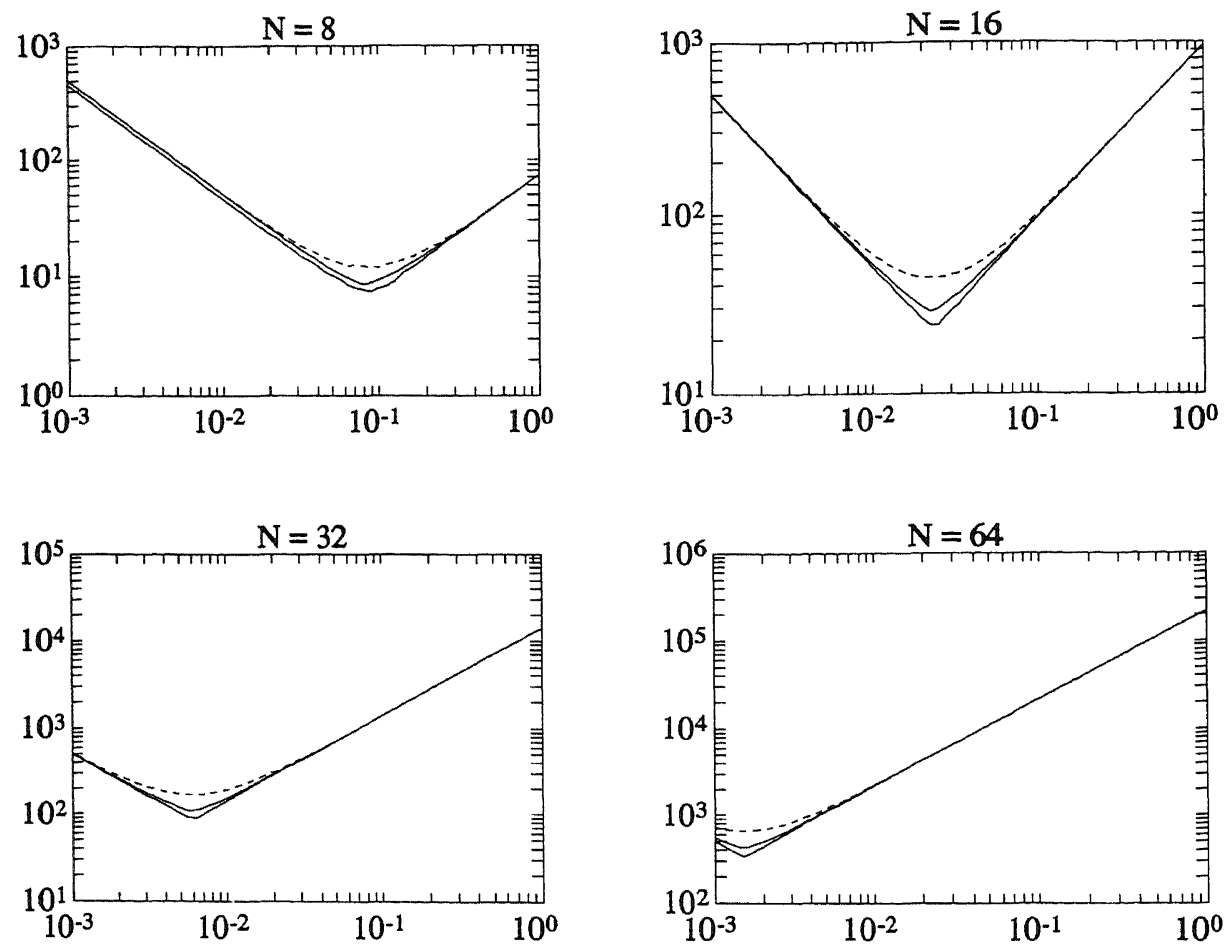

Figure 1. For each graph, $\nu$ is on the horizontal axis. The upper solid curve stands for $r_{1}$, the lower solid curve stands for $r_{2}$ and the dashed curve stands for $r_{3}$.

investigate this step size restriction we note that (3.6) implies that $\alpha_{1}^{2} / \alpha_{2} \leq 1$. Further it follows from [1, subsection 11.4.2] that $\alpha_{1}=O\left(N^{2}\right)$ and $\alpha_{2}=O\left(N^{4}\right)$ so that we arrive at the following corollary.

COROLlary 3.2. For any $\varphi$ satisfying $\varphi(0)=\varphi^{\prime}(0)=1$, there exist constants $C_{1}, C_{2}>0$ such that the process (1.1) is stable if

$$
h \leq\left(\frac{1}{\nu} C_{1}+C_{2} \nu N^{4}\right)^{-1}
$$

We will investigate the sharpness of (3.5a). Denote

$$
r_{2}=\min \left\{r: r \geq 0 \text { and } F_{N}(A) \subset D[-r, r]\right\} .
$$

We have also computed the number

$$
r_{3}=\min \left\{r: r \geq 0 \text { and }\|A+r I\|_{N} \leq r\right\}
$$

so that we are able to compare the requirements of Theorems 2.1 and 2.4 for the example above. The numbers $r_{2}$ and $r_{3}$ have been computed by an algorithm based on bisection. We have used Matlab to perform all our numerical computations. The quantities $r_{1}, r_{2}$ and $r_{3}$ have been computed for $\nu \in[0.001,1]$ 
and $N=8,16,32$ and 64 . The results are displayed in Figure 1. Our experiments illustrate that the estimate (3.5a) is almost sharp. In order to understand the behaviour of $r_{2}$ (as a function of $\nu$ and $N$ ) we note that numerical experiments indicate that $\alpha_{1} \approx 0.08 N^{2}$ and $\alpha_{2} \approx 0.026 N^{4}$ as $N \rightarrow \infty$. When we substitute these relations in (3.5b) we obtain

$$
r_{1} \approx \max \left[0.0032 \nu N^{4}+0.50 / \nu, 0.013 \nu N^{4}+0.12 / \nu\right]
$$

and this approximation becomes better as $N$ becomes larger. For a given constant $\nu$ one may deduce from (3.8) constants $C_{1}$ and $C_{2}$ in (3.7) so as to obtain an a priori step size restriction (necessary and sufficient to apply Theorem 2.4 ) such that (1.1) is stable.

From Figure 1 we conclude that $r_{2}$ and $r_{3}$ are almost equal for most values $\nu$. Therefore our experiments suggest that Theorem 2.1 would yield practically the same threshold value $H>0$ such that (1.1) is stable for $0<h \leq H$. However, actual application of Theorem 2.1 seems much more difficult than applying Theorem 2.4; we have not been able to provide a good upper bound for $r_{3}$.

REMARK 3.3. Theorem 3.1 does not hold if the Legendre-Gauss-Lobatto points are replaced by the Chebyshev-Gauss-Lobatto points $x_{j}=\cos (j \pi / N)$ (the weights $w_{j}$, the inner product (3.3) and quadrature formula (3.4) should be adapted accordingly (see e.g. [1, subsection 2.4])). In fact, numerical experiments show that for some values of $\nu$ and $N$ there are eigenvalues of the matrix $A$ which have positive real parts (see e.g. [10]).

\section{Acknowledgements.}

The authors wish to thank Ch. Lubich and M. N. Spijker for stimulating discussions on the topic of this paper.

\section{REFERENCES}

1. C. Canuto, M. Y. Hussaini, A. Quarteroni, and T. A. Zang, Spectral Methods in Fluid Dynamics, Springer, 1988.

2. J. L. M. van Dorsselaer, J. F. B. M. Kraaijevanger, and M. N. Spijker, Linear stability analysis in the numerical solution of initial value problems, Acta Numerica, 1993 (1993), pp. 199-237.

3. D. Gottlieb, The stability of pseudospectral-Chebyshev methods, Math. Comp., 36 (1981), pp. 107-1 18 .

4. D. Gottlieb and E. Tadmor, The CFL condition for spectral approximations to hyperbolic initial-boundary value problems, Math. Comp., 56 (1991), pp. 565-588.

5. E. Hairer and G. Wanner, Solving Ordinary Differential Equations II, Springer, 1991.

6. H. W. J. Lenferink and M. N. Spijker, A generalization of the numerical range of a matrix, Lin. Alg. Appl., 140 (1990), pp. 251-266.

7. H. W. J. Lenferink and M. N. Spijker, On the use of stability regions in the numerical analysis of initial value problems, Math. Comp., 57 (1991), pp. 221-237.

8. C. Lubich and O. Nevanlinna, On resolvent conditions and stability estimates, BIT, 31 (1991), pp. 293-313.

9. R. P. van der Marel, Stability radius of polynomials occurring in the numerical solution of initial value problems, BIT, 30 (1990), pp. 516-528. 
10. A. Mofid and R. Peyret, Stability of the Chebyshev collocation approximation to the advectiondiffusion equation, Computers Fluids, 22 (1993), pp. 453-465.

11. O. Nevanlinna, Matrix valued versions of a result of von Neumann with an application to time discretization, J. Comp. Appl. Math., 12 \& 13 (1985), pp. 475-489.

12. S. C. Reddy and L. N. Trefethen, Lax-stability of fully discrete spectral methods via stability regions and pseudo-eigenvalues, Comp. Meth. Appl. Mech. Eng., 80 (1990), pp. 147-164.

13. S. C. Reddy and L. N. Trefethen, Stability of the method of lines, Numer. Math., 62 (1992), pp. 235-267.

14. F. Riesz and B. Sz.-Nagy, Leçons d'Analyse Fonctionelle, 2nd ed., Akadémiai Kiado, 1953.

15. B. Sz.-Nagy and C. Foiaş, On certain classes of power-bounded operators in Hilbert space, Acta Sci. Math., 27 (1966), pp. 17-25.

16. B. Sz.-Nagy and C. Foias, Harmonic Analysis of Operators on Hilbert Space, North-Holland Publishing Company and Akadémiai Kiado, 1970. 